\title{
UNIQUENESS PROPERTIES OF SETS WITH BLOCKS ${ }^{1}$
}

\section{A. RUBEL}

1. Introduction. We show in this paper that any set containing appropriately long blocks of consecutive positive integers is a set of uniqueness for certain significant classes of entire functions of exponential type. We study the classes $K(a, c)$ of entire functions $f(z)$ satisfying

$$
f(z)=O(1) \exp (a|x|+c|y|+\epsilon|z|) \text { for each } \epsilon>0 .
$$

If $f(z)$ satisfies (1) but meets the weaker restriction that $f(z)$ is regular for $R(z) \geqq 0$, we shall say that it belongs to $K^{*}(a, c)$. A set $A$ is said to be a set of uniqueness for a class $K$ of functions provided that the only function $f(z)$ in $K$ such that $f(\alpha)=0$ for each $\alpha$ in $A$ is the null function $f(z)=0$. Finally, we say that $A$ has blocks of ratio $\phi$ if there is an increasing sequence $\left[n_{k}\right]_{k=0,1,2,} \ldots$ such that if $n_{k} \leqq n \leqq \phi n_{k}$, then $n$ is contained in $A$. (If $A$ has blocks of ratio $\phi$ for each positive $\phi$, we say that $A$ has blocks of ratio $\infty$.) It is our main purpose to prove the following result.

THEOREM 1. Given $a<\infty$ and $c<\pi$, there is a constant $\phi_{0}=\phi_{0}(a, c)$, depending only on $a$ and $c$, such that every set $A$ with blocks of ratio $\phi>\phi_{0}$ is a set of uniqueness for $K(a, c)$.

The function $\sin \pi z$ explains the significance of the condition $c<\pi$, since $\sin \pi z$ belongs to $K(0, \pi)$, yet vanishes for each positive integer $n$. The minimal character of $\sin \pi z$ is indicated by a celebrated theorem of F. Carlson $\left[1\right.$, p. 153], ${ }^{2}$ which states that the set $I$ of all positive integers is a uniqueness set for each $K(a, c)$ with $a<\infty, c<\pi$. The present work is an extension of Carlson's theorem. It is notable that although Carlson's theorem applies equally well to the larger classes $K^{*}(a, c)$, our Theorem 1 does not. This is shown by the following theorem.

TheOREM 2. There is a fixed set $A$, independent of $a$ and $c$, and hav-

Presented to the Society, October 30,1954 under the title Entire functions and Ostrowski sequences; received by the editors October 19, 1955 and, in revised form, November 17, 1955.

1 The material in this paper constitutes part of a thesis submitted to the University of Wisconsin and prepared under the direction of Professor R. C. Buck. The work was supported under Army Contract No. DA-11-022-ORD-875.

2 Numbers in brackets refer to references at the end of this paper. 
ing blocks of ratio $\phi=\infty$, which fails to be a set of uniqueness for $K^{*}(a, c)$ whatever the numbers $a<\infty$ and $c>0$.

2. Proof of Theorem 1. We shall pretend that we have already found $\phi_{0}$ and will proceed with the proof, in the course of which we shall see how $\phi_{0}$ may be determined. Suppose that the theorem is false. Then there exists a set $A$ with blocks of ratio $\phi>\phi_{0}$ that is not a set of uniqueness for $K(a, c)$. Thus, there is an increasing sequence $\left[n_{k}\right]$ of positive integers, and a function $f(z) \not \equiv 0$ belonging to $K(a, c)$, such that $f(n)=0$ for each $n$ satisfying $n_{k} \leqq n \leqq \phi n_{k}, k=0,1$, $2, \cdots$ We shall derive a contradiction, thus proving the theorem.

Let $g(z)=\sum_{n=0}^{\infty} f(n) z^{n}$. Buck [2] has shown that all of the singular points of $g(z)$, finite and infinite, are contained in the annular sector $D$ whose boundary consists of the concentric circular arcs $|z|=e^{a}$ and $|z|=e^{-a}$ for $|\arg z| \leqq c$, and the radial segments joining them. Put $s_{k}(z)=\sum_{n=0}^{k} f(n) z^{n}$ and $r_{k}(z)=g(z)-s_{k}(z)$. Let $\Gamma_{1}$ be the circle $|z|=R_{1}<e^{-a}$ and let $\Gamma_{2}$ be the contour composed of the circle $|z|=R_{2}>e^{a}$ and some closed curve $C$ which encloses $D$ and which is contained in the annular region $R_{1}<|z|<R_{2}$. Let $\psi$ be the region whose boundary is formed by $\Gamma_{1}$ and $\Gamma_{2}$.

We estimate $\left|r_{n_{k}}(z)\right|$ on $\Gamma_{1}$, making use of the long block of zero coefficients, writing

$$
\left|r_{n_{k}}(z)\right|=\sum_{n \geqq n_{k}} f(n) z^{n}=\sum_{n \geqq \phi n_{k}} f(n) z^{n} .
$$

Hence, for some $\delta_{1}>0$, we have

$$
\log \left|r_{n_{k}}(z)\right| \leqq-\delta_{1} \phi n_{k} \text { for } z \text { on } \Gamma_{1} \text {. }
$$

On $\Gamma_{2}, g(z)$ is bounded, and $s_{n_{k}}(z)$ is a polynomial of degree at most $n_{k}$, so that for some $\delta_{2}$, we have

$$
\log \left|r_{n_{k}}(z)\right| \leqq \delta_{2} n_{k} \text { for } z \text { on } \Gamma_{2} \text {. }
$$

We now choose a convenient circle $\Gamma_{3}$, given by $|z|=R_{3}$, with $R_{2}>R_{3}>e^{a}$, which lies in $\psi$ and encloses $C$. We will use the methods of harmonic measure to estimate $\log \left|r_{n_{k}}(z)\right|$ for $z$ on $\Gamma_{3}$. Let $v_{1}(z)$ and $v_{2}(z)=1-v_{1}(z)$ be the harmonic measures in $\psi$ of $\Gamma_{1}$ and $\Gamma_{2}$ respectively. That is, $v_{1}(z)$ and $v_{2}(z)$ are harmonic in $\psi$, continuous in the closure of $\psi$, and

$$
\left.\left.\begin{array}{ll}
v_{1}(z)=1 \\
v_{2}(z)=0
\end{array}\right\} z \text { on } \Gamma_{1}, \quad \begin{array}{l}
v_{1}(z)=0 \\
v_{2}(z)=1
\end{array}\right\} z \text { on } \Gamma_{2} .
$$

By the familiar two constant theorem, we have, for all $z$ in $\psi$, 


$$
\begin{aligned}
\log \left|r_{n_{k}}(z)\right| & \leqq-\delta_{1} \phi n_{k} v_{1}(z)+\delta_{2} n_{k} v_{2}(z) \\
& =n_{k}\left(-\phi \delta_{1} v_{1}(z)+\delta_{2} v_{2}(z)\right)
\end{aligned}
$$

The choice

$$
\phi_{0}=\left(\delta_{2} / \delta_{1}\right) \max _{z \in \Gamma_{z}} v_{2}(z) / v_{1}(z)
$$

is now apparent. We have

$$
\log \left|r_{n_{k}}(z)\right| \leqq \delta_{1} n_{k}\left(-\phi+\phi_{0}\right) \text { for } z \text { on } \Gamma_{3},
$$

so that $\log \left|r_{n_{k}}(z)\right|$ approaches $-\infty$ uniformly for $z$ on $\Gamma_{3}$. Hence $\lim _{k \rightarrow \infty} s_{n_{k}}(z)=g(z)$ uniformly for $z$ on $\Gamma_{3}$, and therefore for all $z$ within $\Gamma_{3}$. Thus, $g(z)$ is regular throughout the interior of $\Gamma_{3}$. But we have so chosen $\Gamma_{3}$ that it encloses all the singularities of $g(z)$. We conclude, then, that $g(z)$ has no singular points whatever. Hence, $g(z)$ is a constant and we see that $f(n)=0$ for $n=1,2,3, \cdots$. It now follows from Carlson's theorem that $f(z) \equiv 0$, contradicting our hypothesis, thereby completing our proof.

If we make specific choices of $\Gamma_{1}, \Gamma_{2}$, and $\Gamma_{3}$ and determine $v_{1}$ and $v_{2}$, then an explicit estimate of $\phi_{0}$ may be found. A different method for estimating $\phi_{0}$, by means of Carleman's theorem, is essentially outlined in [3]. It leads more easily to an explicit estimate than the present method, but the easier estimate is not as sharp (at least in some special cases) as the present one.

3. Proof of Theorem 2. We now construct a set $A$ with blocks of ratio $\phi=\infty$ which is a set of uniqueness for $K^{*}(a, c)$ for no choice of $a<\infty, c>0$. Let $A$ be the union of the blocks of consecutive integers $n=2^{2^{k}}, 2^{2^{k}}+1,2^{2^{k}}+2, \cdots, 2^{k} 2^{2^{k}}$ for $k=1,2,3, \cdots$. Clearly, $A$ has blocks of ratio $\infty$. By a theorem of Fuchs $[1$, p. 157], we shall have proved our theorem if we can show that

$$
\limsup _{\boldsymbol{r} \rightarrow \infty}[\beta(\boldsymbol{r})-\epsilon \log \boldsymbol{r}]<\infty
$$

for each $\epsilon>0$, where $\beta(r)=\sum_{n \leqq r} 1 / n$, the sum extending only over those $n$ contained in $A$. For any large $r$, we may choose $k$ so that $2^{2^{k}} \leqq r \leqq 2^{2^{k+1}}$. Then

$$
B(r) \leqq \sum_{v=0}^{k} \sum_{n=2^{2^{v}}}^{2^{v} 2^{2^{v}}} 1 / n \sim \sum_{v=0}^{k} \log 2^{v}=O\left(k^{2}\right) .
$$

But $\log r \geqq \log 2^{2^{k}}$. Hence $\beta(r)-\epsilon \log r \leqq O\left(k^{2}\right)-2^{k} \epsilon \log 2$, so that $\beta(r)-\epsilon \log r \rightarrow-\infty$ as $r \rightarrow \infty$, whatever our choice of $\epsilon>0$, so that (2) is certainly valid. This concludes the proof of the theorem. 


\title{
REFERENCES
}

1. R. P. Boas, Jr., Entire functions, New York, 1954.

2. R. C. Buck, A class of entire functions, Duke Math. J. vol. 13, pp. 541-559.

3. L. A. Rubel, Necessary and sufficient conditions for Carlson's theorem on entire functions, Proc. Nat. Acad. Sci. U.S.A. vol. 41, pp. 601-603.

UNIVERSITY OF WISCONSIN AND

CorNell University

\section{ON COMMUTATORS AND JACOBI MATRICES ${ }^{1}$}

\author{
C. R. PUTNAM
}

1. All operators in this paper are bounded linear transformations on a Hilbert space. The commutator $C$ of two operators $A$ and $B$ is defined by

$$
C=A B-B A .
$$

The closure, $W$, of the set of values $(C x, x)$ when $\|x\|=1$ is a closed convex set (Hausdorff, cf. [8, p. 34]). A complex number $z$ will be said to belong to the interior of $W$ if $z$ is in $W$ and if one of the following conditions holds: (i) If $W$ is two-dimensional, then $z$ does not lie on the boundary of $W$; (ii) If $W$ is a line segment, then $z$ is not an end point; (iii) $W$ consists of $z$ alone.

It was shown in [4] that if $A$ (or $B$ ) is normal, or even semi-normal, so that $A A^{*}-A^{*} A$ is semi-definite, then 0 belongs to $W$, but is not necessarily in the interior of $W$. (That, for arbitrary $A$ and $B$, in general 0 need not even belong to $W$ was shown in [2].) In fact, if $A=\left(a_{i j}\right)$ is defined by $a_{i, i+1}=1, a_{i j}=0$ if $j \neq i+1$, then $C=A A^{*}-A^{*} A$ $=\left(c_{i j}\right)$ is the self-adjoint matrix all elements of which are zero except $c_{11}=1$. Consequently, $C \geqq 0$ with a spectrum consisting of $\lambda=0,1$; hence $W$ is the segment $0 \leqq \lambda \leqq 1$ and 0 is not in the interior of $W$. Moreover, the above $C$ can also be expressed by $C=D A^{*}-A^{*} D$ where $D$ is the (self-adjoint) Jacobi matrix $A+A^{*}$; cf. $\S 5$ below.

The problem to be considered in the present paper is that of determining a sufficient condition guaranteeing that 0 belongs to the interior of $W$. Such a condition yields information concerning the spec-

Received by the editors November 25, 1955.

1 This work was supported by the National Science Foundation research grant NSF-G-481. 